\title{
Design and implementation of a wireless sensor communication system with low power consumption for energy harvesting technology
}

\author{
Vlad Marsic, Meiling Zhu, Member, IEEE, and Stewart Williams
}

\begin{abstract}
This paper presents the design and implementation of a wireless sensor communication system with a low power consumption for integration with energy harvesting technology, that can be employed in energy autonomous wireless sensor communication applications. The design and implementation focus on three levels: hardware, software and data transmission. The resulted system is able to satisfy all the theoretical and practical requirements in order to be included in a wireless sensor structure that is able to give the device self-powered autonomy, due to a smart inter-correlated management of the energy resources.
\end{abstract}

Keywords - energy harvesting, low power consumption, wireless sensor communication system.

\section{INTRODUCTION}

$\mathrm{S}$ UCCESSFUL high technology manufacturing industries are offering a range of high value products and services. High value products are technology intensive, expensive and reliability critical requiring engineering services (e.g. maintenance and repair) throughout the life cycle, e.g. railway axle bearings, wind turbine rigs, and aircraft engines and wings. These produces must be monitored and assessed throughout their lifetime. Currently, the process is often manual, expert dependent and time consuming, and often lacks information on what is going on while in active service. High technology industries are increasingly demanding easy access to information for better decision-making in the maintenance process over the life cycles of their products. The management process typically requires an autonomous monitoring system like the one proposed in this research program, that operates as long as possible to collect, elaborate, store and share information within communication sensor networks powered by energy harvesting technology, rather than powered by mains electricity or batteries.

Energy harvesting means to scavenge ambient energy from human motion, machine vibration, thermal and solar energy, and then convert the scavenged energy into useable electrical energy to provide electrical power for small electronic and electrical devices making them self-

Vlad Marsic is with the School of Applied Sciences, University of Cranfield, United Kingdom (e-mail: V.A.Marsic@cranfield.ac.uk).

Meiling Zhu is with the School of Applied Sciences, University of Cranfield, United Kingdom (e-mail: M.Zhu@cranfield.ac.uk).

Stewart Williams is with the School of Applied Sciences, University of Cranfield, United Kingdom (e-mail: S.Williams@cranfield.ac.uk) sufficient in energy supply. Benefits of this technology include: no need of battery replacement, no need of cabling, easy to retrofit infrastructures, and a "truly fitand-forget" approach that allows reduction of physical installation, replacement and maintenance costs, and time. Because of the limited amount of energy harvested by vibrating harvesters, the majority of the actual technology is currently using as energy sources the light, air and water movement to power the Wireless $\underline{\text { Sensor Networks }}$ (WSN). These systems can monitor processes in open spaces, but cannot be applied inside buildings or other structures operating in a full enclosure habitat. There is an increasing demand for self-powered WSNs capable to operate without light, water or air movement in a partially or fully closed environment. The best efficiency in such conditions is provided by the vibration harvester, which does not require a special structure and positioning in the monitored environment like the Radio Frequency (RF) or thermal harvesters ([1], [2]).

This paper presents the design and implementation of a wireless sensor communication system that achieves low power consumption at three levels, and can be integrated with energy harvesting technology for energy autonomous wireless sensor communication applications.

A self-powered autonomous wireless sensor system is composed of a power source and a wireless communication system. These two main components can be also divided into five functional blocks: Vibration Energy Harvester Block, Power Management Block, Wireless Communication Block, Wireless Sensor Management Block and Sensors Block.

The Vibration Energy Harvester Block represents the power supply. The energy it produces is directly dependent on the vibration strength and the frequency provided by the wireless sensor deployment environment.

The Power Management Block extracts, transforms and stores the energy generated by the vibration harvester, making it useful as a stabile DC power source.

The Wireless Sensor Management Block takes data from the Sensors Block and processes it in order to be sent to the Wireless Communication Block.

The Sensors Block interacts with the external environment and interprets specific stimuli (e.g. temperature, acceleration, light intensity, pressure, etc.) by transforming them in electrical signals.

The Wireless Communication Block transmits the 


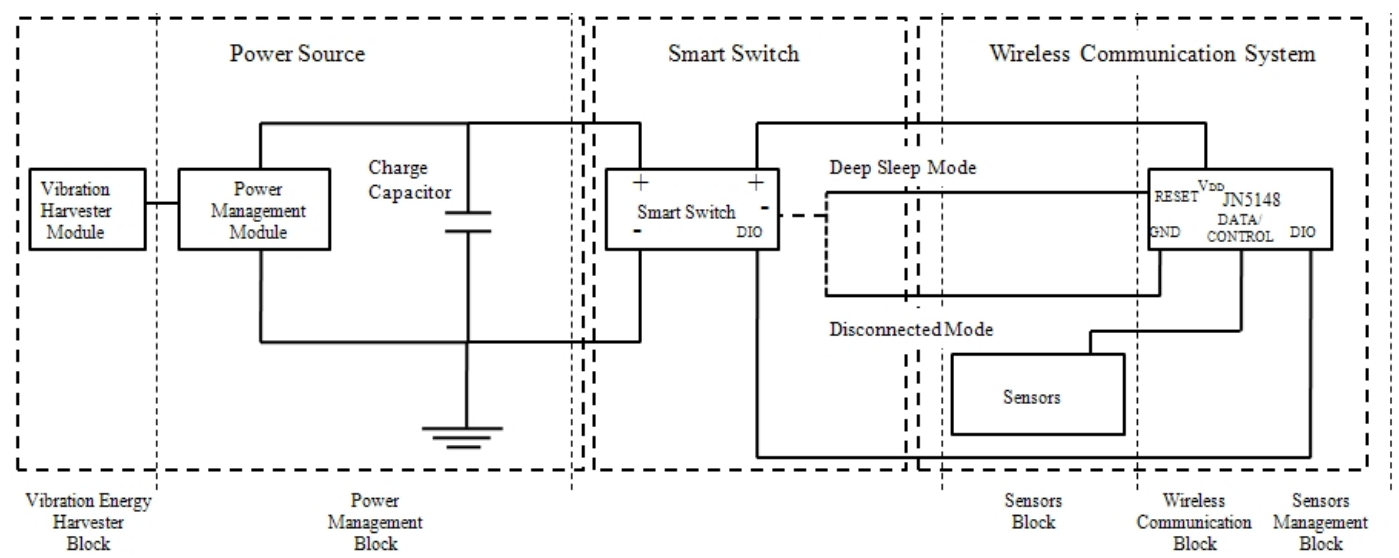

Fig. 1. System Design and Implementation at Hardware Level

data provided by the Wireless Sensor Management Block, by air to a base station which can be linked to the Internet or directly to the user.

This paper will discuss the design and implementation of the wireless communication system which must satisfy certain conditions for achieving energy autonomy.

\section{SySTEM DESIGN}

Considering the power consumed by the transceiver/receiver $(\mathrm{Tx} / \mathrm{Rx})$ in the sleep and active modes, the speed of the Central Processing Unit (CPU), the dimension of Random $\underline{\text { Access }}$ Memory (RAM), and of the Flash Memory (Flash), the system JN5148, offered by Jennic (NXP Semiconductors) satisfies the requirements for a low power wireless communication system.

JN5148 is an ultra low power, high performance wireless microcontroller. The device features an enhanced 32-bit Reduced Instruction $\underline{\text { Set }}$ Computing (RISC) processor with programmable clock speeds. It also includes a 2.4GHz IEEE802.15.4 compliant transceiver, with a power consumption of $15[\mathrm{~mA}]$ when transmitting, $17.5[\mathrm{~mA}]$ when receiving, and $1.25[\mu \mathrm{A}]$ in sleep mode, $128 \mathrm{kB}$ RAM, and a rich mix of analogue and digital peripherals. The $4 \mathrm{Mb}$ flash memory footprint allows the device to run both a network stack (e.g. ZigBee PRO) and an embedded application.

Having established the core structure (JN5148) of the wireless communication system, we must focus on the next stage of system design. The system is designed and implemented at three levels: hardware, software and data transmission. The problems to be resolved are:

- Reducing the power consumption in the active mode (while executing the programmed instructions, reading sensors' data, and transmitting wirelessly the information), and in the passive mode (while the device is disconnected from the power source or enters an idle mode known as deep sleep, until the energy storage device regains enough energy for the system to function in active mode);

- Preserve the system's basic functionality and compatibility with standards after reducing the energy consumption (i.e. preserve the main function of transmitting data using a certified protocol like IEEE
802.15.4, and respect the restrictions involving power and license for certain geographic regions).

\section{SYSTEM IMPLEMENTATION}

The system is implemented at three levels: hardware, software and data transmission. The system implementation at each of these three levels is presented in detail below.

\section{A. Hardware level}

As already mentioned, the system is powered using a vibration harvester which converts the vibration energy from the surrounding environment in alternative current and voltage. This output is afterwards transformed by the Power Management module in Direct Current (DC). The amount of energy supplied to the wireless communication system is influenced (e.g. reduced, discontinued) in direct proportion with the surface of the harvester, the strength, frequency and period of its vibration. Due to this fact, the system functions in low duty cycles, to conserve its energy. When enough energy is available, the system reads the sensors, transmits the data wirelessly to the user, and then enters in a low energy consumption state (sleep mode or even disconnected from the power supply). The low energy state can be in the range of seconds or hours, and depends on how fast the harvester can recharge a capacitor up to a certain level necessary (Smart Switch 'ON' Voltage) to allow the system to function again.

The low energy state can reach the minimum of power consumption in two scenarios: either the system enters in deep sleep mode and can be "woken up" by an external monitoring circuit, or the system is disconnected from the power supply by an external monitoring circuit. In both cases, a smart switch is mandatory in order to control and monitor the low energy state of the system until the available energy allows the next active stage (Fig. 1).

The charging element from the Power Source Block can be a capacitor.

The smart switch required to obtain the low energy state can be connected for the deep sleep mode to the RESET pin of JN5148, or for the disconnected mode to the gate of the N-MOS transistor Q2. The smart switch operates in two stages: 
1. When the voltage on the charging element reaches the threshold voltage of the Integrated Circuit $\underline{\text { Supervisor }}$ (ICS), the RESET pin of the ICS, after a certain delay time, is set to Active, which will open the connection to the ground for JN5148 or will wake up the JN5148 module from the deep sleep mode.

2. Afterwards the wireless microcontroller starts the booting sequence. After booting, the JN5148 sets to Low logic the Digital Input-Output (DIO) connected to the gate of N-MOS transistor Q1, disconnects the ICS, read sensors, transmits the data, sets to High logic the same DIO as before, and reconnects the ICS. This will detect if the voltage on the charging element is below his threshold voltage, and will give a Passive State signal to the gate of N-MOS Q2, opening the transistor's high impedance state, and cutting the power for the disconnected mode.

In order to choose the best way to define the circuit's low energy state, the first step is to determine the values associated with the deep sleep mode of the JN5148 wireless module.

\section{Testing and Measurements}

The measurements were made in three settings using an approach similar to the one described in [3].

The first board for testing the current consumption for JN5148 was the sensor board DR1048. For the deep sleep mode measurements, the sensor board, which contains the wireless communication module JN5148, was directly connected to the testing set.

The second board for measuring the current consumption corresponding to the wireless module was built on a single layer cooper prototype board, using the minimum configuration for low power applications suggested in the following Jennic documentation articles: DR1048_Schematic, JN-AN-1146 and JN-AN-1055.

The last setting for measuring the current consumption in deep sleep mode for the JN5148 module was made using the same one layer cooper testing board, but replacing the $18 \mathrm{~K} \Omega$ resistor $(\mathrm{R})$ between the Reset pin and the Vcc with a $10 \mathrm{M} \Omega$ pull-up resistor.

The results quantifying JN5148's current consumption in deep sleep mode are included in Table I.

TABLE 1: CURRENT CONSUMPTION MEASUREMENTS

\begin{tabular}{|l|l|}
\hline Measuring setting & Deep sleep mode current consumption $[\boldsymbol{\mu} \boldsymbol{A}]$ \\
\hline DR1048 sensor board & 1598.41 \\
\hline Prototype board $(18 \mathrm{~K} \Omega)$ & 258.32 \\
\hline Prototype board $(10 \mathrm{~K} \Omega)$ & 9.58 \\
\hline
\end{tabular}

To determine the current consumption for the disconnected mode, a smart switch was introduced, and the sensor board which contains the wireless communication module JN5148 was connected to this switch.

When choosing the ICS, three elements were taken into consideration: threshold voltage, current consumption, and the time delay before the Reset pin changes its state. The smart switch design that we implemented after testing more than 20 ICSs different in their output signal characteristics contains the MCP121-315E Voltage Supervisor (Active-low, $1.75 \mu \mathrm{A}$ current consumption and time delay of $120 \mathrm{~ms}$ ).

The disconnected mode is provided by the implementation of the smart switch. It was measured for current consumption at maximum voltage for the switch to remain closed. The switch was connected with the JN5148 wireless module to the DR1048 sensor board.

The current consumption measurements in disconnected mode are the same for all measuring settings, i.e. $1.58 \mu \mathrm{A}$.

Considering the results measured for the circuit's low energy state, and knowing that for the deep sleep mode another external circuit needs to be added to "wake-up" the module, thus adding another consumer besides the wireless module, the implementation of the smart switch not only resolves the issue of energy consumption during long inactivity periods, but can also be applied to other wireless modules on the market.

\section{B. Software level}

To reduce the energy consumption at software level, one requires a good practical approximation and calculation of the energy used in order to boot the system, read the sensors and wirelessly transmit the data.

The optimisations that can be addressed at the software level are concerned with reducing to minimum the dimensions of the program, accelerating the operations, eliminating the unnecessary delays, and reducing the time of the active mode.

Considering the worst case scenario in which a charging element of minimum capacity can be filled up in the maximum accepted period of time, and the best case

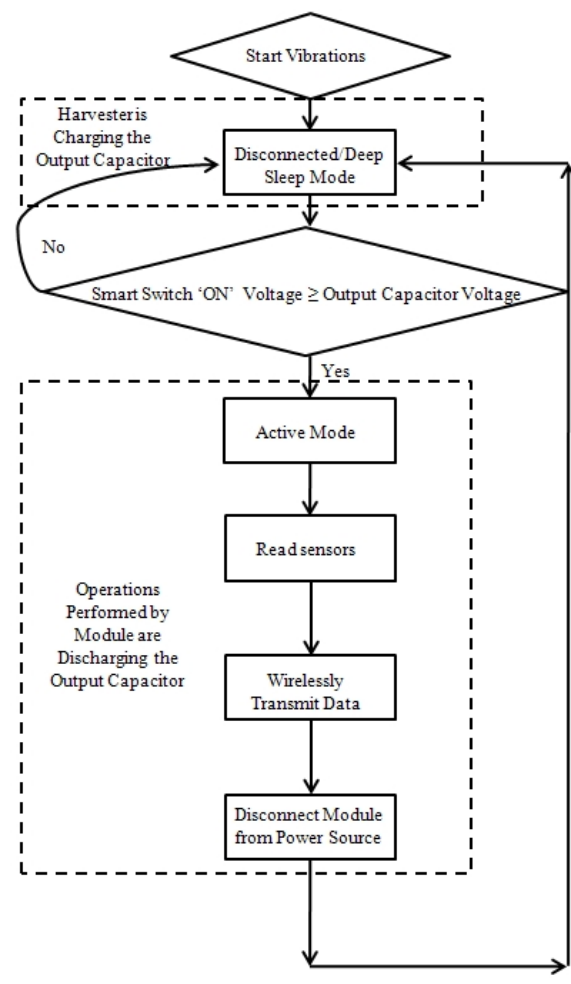

Fig. 2. Second software algorithm 
scenario in which a charging element of maximum capacity can be charged in the minimum period of time, we designed two algorithms that capture the system functionality when addressing these scenarios (Fig. 2 captures the second algorithm). We implemented both algorithms in application programming. The first one has the advantage to be adaptive when a random minimum power is provided to the charging element, but its main disadvantage is the impossibility of real timing for reading the sensors. The second one has the advantage of transmitting the data immediately after reading the sensors, thus eliminating the problem of real timing, but it does not have the capacity to adapt when the charging element is confronted with random variable power.

Both these disadvantages are corrected and compensated:

- The timing issue is solved at the next stage of system design, data transmission, by associating to each node a time allocation frame in addition to its data frame, so that the timings can be correlated and errors reduced.

- The adaptation problem is addressed by reducing the code size and the start-up time of the application with functions from the Low Power Transmit library (libLPTI.a) for the IEEE 802.15.4 stack (provided by the Jennic programming team).

\section{Data transmission level}

At this level, the ideal scenario offering the lowest power consumption would be the Self-Powered Autonomous Wireless Sensor offering the same functionality as a passive $\underline{\text { Radio }}$ Frequency IDentification (RFID) tag. In real life, this functionality implies, except for the low consumption part, only downsides. On the one side, the distance between the RFID tag and the reader would be constrained by the fact that local standards regulate the maximum power level for transmitting, and with such power level one cannot transmit further than a certain short distance. On the other side, given this type of functionality, the power would be very low and insufficient to implement an algorithm for communication and data transmission with multiple sensors.

Existing attempts to combine the IEEE 802.15.4 compliant wireless networks with RFID technology in a hybrid dual transceiver/receiver are suitable only for battery powered systems, due to the amount of power required. However, at the present moment, mixing two different transceivers/receivers (one for the IEEE 802.15.4/ZigBee protocol, and the other one for the Amplitude Modulation (AM) used by RFID technology, [4]) into the same product, increases the expenses and complicates the structure of the wireless network.

The ZigBee protocol approach is not yet technologically suitable for this project, because it implies the presence of routers which are devices continuously powered to connect the end device nodes to the base station, without being allowed to have a low power duty cycle.

A ZigBee network without routers could be resembled to a star network configuration, in which every node transmits and receives data to and from the base station.
This configuration is identical with the IEEE 802.15.4 star configuration. Therefore, the IEEE 802.2.15.4 protocol suits better the purposes of this work when compared to ZigBee, as it offers a simplification of the software and data transmission levels, thus resulting in less power consumption.

Due to these considerations, we have chosen for the data transmission level an IEEE 802.15.4 star configuration. The wireless sensors representing the nodes of the network use a IEEE 802.15.4 minimum data frame format, less than 32 bytes per total length, to transmit the data they collect to the base station. The transmission uses 3 communication channels with a dynamic calculated time allocation frame in order to prevent data collision between nodes, given the fact that all nodes only transmit data, without performing $\underline{\text { Clear }}$ Channel Asssessment (CCA), Energy Detection (ED), or receiving data from the base station. These limitations were imposed in order to save energy. The type of the base station can be Simple (one station), or Multiple Access (multiple fixed stations interconnected with each other, offering better reception and efficiency) (Fig. 3).

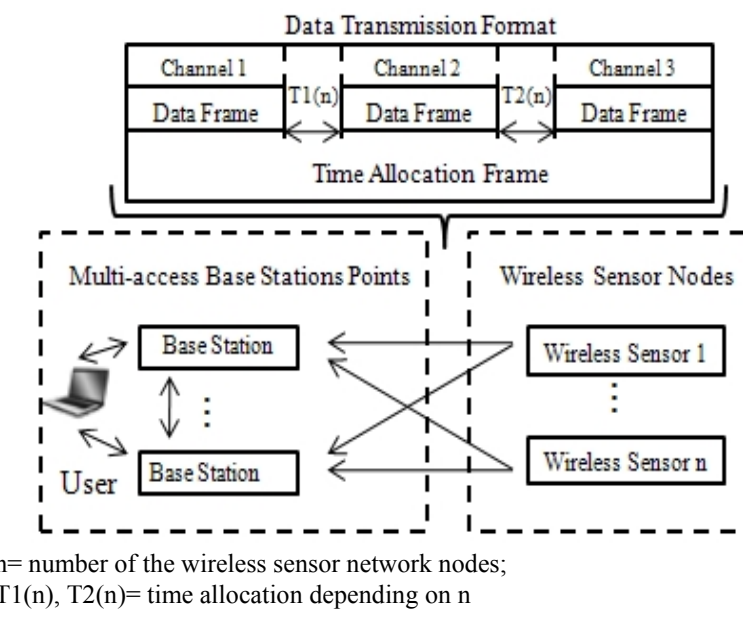

Fig. 3. Modified Network Topology for the New Data Frame Format in Multichannel Communication

\section{CONCLUSIONS}

The present paper describes at all three levels hardware, software, and data transmission - the design and implementation of a wireless communication system suitable for all types of ultra low vibration energy harvesters, by taking into consideration the present standards, protocols and electronic technology offers.

\section{REFERENCES}

[1] Energy Autonomous Systems: Future Trends in Devices, Technology, and Systems, CATRENE Working Group on Energy Autonomous Systems, 2009.

[2] CSEM Scientific and Technical Report 2009, CSEM Centre Suisse d'Electronique et de Microtechnique SA, 2009.

[3] Eduardo Casilari, Jose M. Cano-García and Gonzalo CamposGarrido, "Modelling of Current Consumption in 802.15.4/ZigBee Sensor Motes", Sensors, vol. 10, pp. 5443-5468, Jun. 2010.

[4] P. Medagliani, G. Ferrari, M. Marastoni, "Hybrid ZigBee-RFID Network With Highest Energy Efficiency", in Proc. of the $3^{\text {rd }}$ International Workshop on Distributed Cooperative Laboratories, Italy, 2008. 